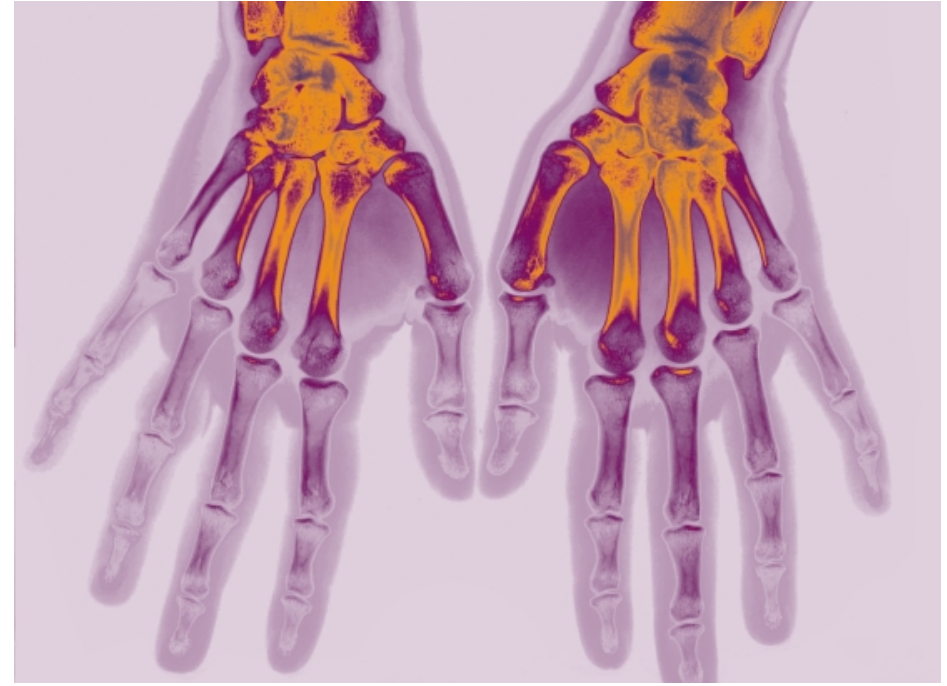

GENE THERAPY

\section{Inflammation blockade}

Rheumatoid arthritis (RA) is a disabling autoimmune disease of the synovial joints, characterized by chronic inflammation and the destruction of cartilage and bone. Proinflammatory cytokines, such as tumour-necrosis factor- $\alpha$ (TNF$\alpha$ ) and interleukin 6 (IL-6), have been implicated in the pathogenesis of this disease. Recent work by Shouda and colleagues in The Journal of Clinical Investigation shows that blocking cytokine signalling by inducing the expression of the cytokine signal regulator SOC3/CIS3 (suppressor of cytokine signalling $3 /$ cytokineinducible $\mathrm{SH} 2$ protein 3 ) effectively reduces bone destruction in mouse autoimmune arthritis, thus representing a possible new therapeutic target.

Earlier work, both in vitro and in vivo, established a role for IL-6 in the development of arthritis. IL- 6 acts by activating Janus kinase (JAK) tyrosine kinases and the transcription factor STAT3(signal transducer and activator of transcription 3. SOCS3 was previously cloned by Shouda's group, and was shown to inhibit JAK kinases and to negatively regulate STAT3 functions. As SOCS 3 is strongly induced by IL- 6 , the authors decided to investigate whether SOCS3 plays a negative regulatory role in the progression of RA.

To investigate the importance of STAT3 activation and SOCS3 induction in RA, adenoviral transfer was used to overexpress SOCS3 or a dominant negative form of STAT3 (dnSTAT3) in synoviocytes isolated from patients with RA. The proliferation of cells infected with either of these constructs was significantly reduced, as was the production of IL- 6 and TNF- $\alpha$. These results show that the proliferation and cytokine production of RA-synoviocytes in vitro is dependent on JAK/STAT3 signalling and that these processes can be inhibited by the expression of SOCS3 or dnSTAT3.

Do these constructs have any effect on the development of arthritis in vivo? Shouda and coworkers injected SOCS3 and dnSTAT3 adenovirus into the ankle joints of mice prone to antigen-induced arthritis (AIA) or collagen-induced arthritis (CIA). In the AIA model, both dnSTAT3 and CIS3 drastically reduced the severity of arthritis and joint swelling compared with control animals. However, in the CIA model the dnSTAT3 adenovirus was less effective than SOCS3, which significantly reduced the severity of arthritis. Finally, the SOCS3 adenovirus was also shown to be an effective treatment for established arthritis.

The authors conclude that adenovirus-mediated gene transfer of the SOCS3 gene could represent a new approach for effectively blocking the pathogenesis of RA. Jenny Buckland

(9) References and links ORIGINAL RESEARCH PAPER Shouda, T. et al Induction of the cytokine signal regulator
Intion Induction of the cytokine signal regulator
SOCS3/CIS3 as a therapeutic strategy for treating inflammatory arthritis. J. Clin. Invest. 108 1781-1788 (2001)

FURTHER READING Feldmann, M. et al. Cytokine blockade in rheumatoid arthritis. $A d v$. Exp. Med. Biol. 490,119-127 (2001)

\section{IN BRIEF}

\author{
AUTOIMMUNITY
}

Association of BAFF/BLyS overproduction and altered B-cell differentiation with Sjögren's syndrome.

Groom, J. et al. J. Clin. Invest. 109, 59-68 (2002).

Sjögren's syndrome (SS) is a chronic autoimmune disorder that causes a dry mouth and eyes. It is associated with B-cell hyperactivity and serum autoantibodies. BAFF-transgenic mice develop a lupus-like disorder. This paper shows that ageing BAFFTg mice develop a pathology that resembles SS. Marginal-zonelike B cells were identified in salivary glands of BAFF-Tg mice. Humans with SS have elevated levels of circulating BAFF, perhaps because of an imbalance in BAFF production.

\section{INNATE IMMUNITY}

Human macrophage activation programmes induced by bacterial pathogens.

G. J. Nau et al. Proc. Natl Acad. Sci. USA 22nd January 2002 (epub ahead of print).

Although the innate immune system is not 'specific', there are indications that it can differentiate between different classes of bacteria. Here, the responses of macrophages to three classes of bacteria (Gram-negative, Gram-positive and mycobacteria) were compared using genome-wide gene expression profiling. A common activation programme of gene expression (132 induced and 59 repressed genes) was identified. Pathogen-specific responses were also identified, such as the repression of IL- 12 by Mycobacterium tuberculosis.

\section{CYTOKINE SIGNALLING}

Physical and functional interaction between GATA-3 and Smad3 allows TGF- $\beta$ regulation of GATA target genes.

Blokzijl, A. et al. Curr. Biol. 12, 35-45

GATA-3 is a master transcriptional activator of $\mathrm{CD}^{+} \mathrm{T}_{\mathrm{H}}$ 2-cell differentiation. TGF- $\beta$ has complex effects on T-cell differentiation and has been proposed to inhibit $\mathrm{T}_{\mathrm{H}} 2$ differentiation by inhibiting GATA-3 production. Here, Blokzijl, A. et al. reveal a new interface between TGF- $\beta$ and GATA-3. They show that smad 3 (a crucial TGF- $\beta$ signal transducer) interacts physically with GATA- 3 to form a complex that can regulate the transcription of $\mathrm{T}_{\mathrm{H}} 2$ cytokine genes.

\section{IMMUNE REGULATION}

\section{$\mathrm{T}_{\mathrm{H}} 2$ response induction by dendritic cells: a role for CD40}

MacDonald, A.S. et al. J. Immunol. 168, 537-540 2002

Dendritic cells are thought to be key regulators of $\mathrm{T}_{\mathrm{H}} 1-\mathrm{T}_{\mathrm{H}} 2$ differentiation during $\mathrm{CD} 4^{+} \mathrm{T}$-cell priming. They interpret microbial signals and relay them to $\mathrm{T}$ cells through accessory molecules (such as CD40). However, most work has focused on the induction of $\mathrm{T}_{\mathrm{H}} 1$ cells. By adoptive transfer of CD40-deficient dendritic cells that have been primed with $\mathrm{T}_{\mathrm{H}} 1$ - or $\mathrm{T}_{\mathrm{H}} 2$-inducing microbial stimuli, MacDonald et al. shows a crucial role for CD40CD40L interactions in the induction of $\mathrm{T}_{\mathrm{H}} 2$, but not $\mathrm{T}_{\mathrm{H}} 1$, responses. 


\section{IN BRIEF}

\section{ALLERGY}

The absence of interleukin 9 affects neither the development of allergen-induced pulmonary inflammation nor airway hyper-reactivity.

McMillan, S. J. etal. J. Exp. Med. 195, 51-57 (2002).

The $\mathrm{T}_{\mathrm{H}}$ 2-derived cytokine IL-9 has been implicated in the development of asthma. McMillan et al. investigated the effect of IL-9 deficiency on the development of asthma and airway hyperreactivity (AHR) after allergen challenge. AHR and eosinophilia occurred to a similar degree in $I l 9^{-1-}$ mice and wild-type mice, and goblet cell hyperplasia and immunoglobulin E production were also unaffected in the absence of IL-9. IL-9 is therefore not obligatory for asthma development.

\section{T-CELL HOMEOSTASIS}

Homeostatic competition between T cells revealed by conditional inactivation of the mouse Cd4 gene.

Wang, Q. et al. J. Exp. Med. 194, 1721-1730 (2002).

The role of T-cell receptor (TCR) signalling in T-cell homeostasis and post-thymic selection of naive T cells is unknown. Here, Wang et al. report the impairment of TCR signalling by conditionally inactivating expression of the co-receptor CD4 and the effects this has on homeostatic events. They show that $\mathrm{T}$ cells compete with each other during homeostatic proliferation and that T cells lacking $\mathrm{CD} 4$ compete poorly, indicating that the competition is based on the strength of TCR signal the cells receive.

\section{HAEMATOPOIESIS}

\section{Identification of the earliest prethymic bipotent T/NK} progenitor in murine foetal liver.

Douagi, l. et al. Blood 99, 463-471 (2002).

Douagi et al. describe the identification of a novel population of common T/NK cell progenitors (C-TNKPs) within the foetal liver. These cells are proposed to represent the immediate developmental step before migration to the thymus. This population of $\mathrm{B} 220^{\text {lo }}$ c-kit ${ }^{+} \mathrm{CD} 19^{-}$cells, which compose $0.2 \%$ of foetal liver cells, represent $70 \%$ of T-cell precursors in the foetal liver. These cells can produce both $\mathrm{T}$ - and NK-cell progeny at the single cell level and are present in athymic mice, indicating their prethymic origin.

\section{IMMUNE REGULATION}

\section{IL-10-producing CD4+ ${ }^{+}$T cells mediate tumour rejection.}

Segal, B. M., Glass, D. B. \& Shevach, E. M. J. Exp. Med. 168, 1-4 (2002).

Although IL-10 is typically thought to have immunosuppressive functions, Segal et al. describe a pro-inflammatory population of IL-10-producing $\mathrm{CD} 4^{+} \mathrm{T}$ cells that mediate anti-tumour immunity. In a subcutaneous model of glioma cell growth, IL-10producing $\mathrm{CD} 4^{+} \mathrm{T}$ cells that were crucial for tumour rejection were generated after vaccination with irradiated glioma cells. The cells had a cytokine profile resembling the immunoregulatory $\mathrm{T}_{\mathrm{R}} 1$ cells that can be generated in vivo in the presence of IL-10.

\section{Stop!}

Chronic infection with hepatitis $\mathrm{C}$ virus (HCV) is one of the main causes of liver disease, with about 170 million people infected worldwide. The mechanisms which $\mathrm{HCV}$ employs to promote chronic infection remain poorly understood - partly because of the lack of an in vitro culture system and small animal models - although active evasion of the immune response is a strong possibility. Two recent papers in the Journal of Experimental Medicine now show that crosslinking of CD81 by the HCV envelope protein E2 can inhibit natural killer (NK) cell function, which might affect the innate immune response to $\mathrm{HCV}$ and promote viral persistence.

CD81 is a member of the tetraspanin family of proteins, which are components of large molecular complexes that act as cell-surface organizers coupling different cellular functions. HCV-E2 has previously been shown to bind to the main extracellular loop of CD81. In the current studies, the functional consequences of this interaction for NK cells were investigated. Interferon- $\gamma($ IFN- $\gamma)$ production in response to NK-cell stimulation by the cytokines IL-2, IL-12 or IL-15 was inhibited by crosslinking of CD81. Both groups then induced NK-cell activation by crosslinking CD16 - the lowaffinity immunoglobulin $\mathrm{G}$ receptor (FC $\gamma \mathrm{RIII}$ ), which is one of the main activation receptors on NK cells. Crosslinking of CD81 on the NK cells by HCV-E2 or anti-CD81 antibodies inhibited CD16-induced tumour necrosis factor- $\alpha$ (TNF- $\alpha)$ and IFN- $\gamma$ production. A similar inhibitory effect was observed for expression of the NK-cell activation marker CD25 and the release of cytotoxic granules. When the effect of CD81 crosslinking on T cells and NK cells was compared, both groups observed a co-stimulatory effect on T cells but an inhibitory effect on NK cells.

Protein phosphorylation by activated protein tyrosine kinases is an important consequence of NK-cell activation by engagement of CD16. So, what is the effect of CD81 crosslinking on these signalling events? Crotta and colleagues found that CD81 crosslinking inhibited tyrosine phosphorylation events, including the phosphorylation of $\mathrm{CD} 3 \zeta$ and ERK2, specific substrates that are known to be phosphorylated following NK-cell activation.

What is the mechanism by which CD81 exerts its inhibitory function on NK cells? NK-cell activation is determined by the balance between engagement of stimulatory and inhibitory receptors. To investigate potential interactions with killer inhibitory receptors and the inhibitory signalling phosphatases SHP1 and SHP2, coimmunoprecipitation experiments were performed. No specific interaction was detected, so CD81 seems to mediate inhibition using a previously unrecognized, and as yet undefined, negative signalling pathway.

The results from these studies define an efficient HCV immune evasion strategy and show that the interaction of HCV-E2 with CD81 on NK cells provides a 'stop' signal that blocks NK-cell activation. It is likely that the ability of HCV to interfere with NK function and affect the early phase of the immune response contributes to its success at establishing chronic infections in the host.

Elaine Bell

(D) References and links

ORIGINAL RESEARCH PAPERS Crotta, S. et al. Inhibition of natural killer cells through engagement of CD81 by the major hepatitis C virus envelope protein. J. Exp. Med. 195, 35-41 (2002) | Tseng, C.-T. K. \& Klimpel, G. R. Binding of the hepatitis $C$ virus envelope protein E2 to CD81 inhibits natural killer cell functions. J. Exp. Med. 195, 43-49 (2002)

FURTHER READING Cohen, J. The scientific challenge of hepatitis C. Science 285, 26-30 (1999) WEB SITE

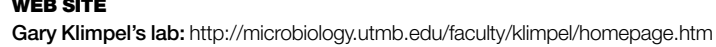

